\title{
Quadratures with Remainders of Minimum Norm. II
}

\author{
By R. E. Barnhill* and J. A. Wixom
}

1. Introduction. Let the quadrature remainder with $n$ base points be given by

$$
R_{n}(f)=\int_{-1}^{1} f-\sum_{k=1}^{n} A_{k} f\left(z_{k}\right)
$$

where $f$ is in the Hilbert space $L^{2}\left(E_{\rho}\right) . L^{2}\left(E_{\rho}\right)$ is $\{f(z): f$ is analytic inside the ellipse $E_{\rho}$ and $\iint_{E_{\rho}}|f(z)|^{2} d x d y$ exists $\}$, where $E_{\rho}$ is the ellipse with foci at \pm 1 , semimajor axis $a$, semiminor axis $b=\left(a^{2}-1\right)^{1 / 2}$ and $\rho=(a+b)^{2}$, and the double integral is taken over the region inside the ellipse. For additional information on the space $L^{2}\left(E_{\rho}\right)$ the reader is referred to Davis [5]. For fixed $n, R_{n}$ is a bounded linear functional on $L^{2}\left(E_{\rho}\right)$. The problem is to minimize $\left\|R_{n}\right\|=\sup \left(\left|R_{n}(f)\right| /|| f||\right)$ by an appropriate choice of the $A_{k}$ and $z_{k}$ in Eq. (1). In [2] the problem of minimizing || $R_{n} \|$ with respect to the $A_{k}$ was solved, and this paper extends those results to the case of variable base points $z_{k}$.

The idea of minimizing the norm of the remainder has appeared in several papers. For the Hardy space $\mathrm{H}_{2}$, Yanagihara [9] posed it for 2-, 3- and 4-point quadrature rules and obtained explicit solutions for the weights and points. The first author rediscovered some of Yanagihara's results and also solved the minimization problem for the space $L^{2}\left(E_{\rho}\right)$ in his doctoral dissertation [10]. Valentin extended some of Yanagihara's results for the space $H_{2}$ and he also considered the space $L^{2}(R)$ ( $R$ being the unit disc) in his doctoral dissertation [8]. For the space $H_{2}$, Wilf [11] has also considered this problem. In the latter three papers the cases solved were done numerically. The problem is also mentioned in Davis [12].

2. Minimization of the Norm of the Remainder. For an arbitrary normed linear space $X$, it is difficult to find a representation of $\left\|R_{n}\right\|$ that can be computed. However, since $L^{2}\left(E_{\rho}\right)$ is a Hilbert space, the Riesz representation theorem for Hilbert space can be used to find a computable representation of $\left\|R_{n}\right\|$. This idea was first applied to quadratures by Davis [3]. Specifically, if $\left\{P_{m}(z)\right\}_{m=0}^{\infty}$ is a complete orthonormal sequence in $L^{2}\left(E_{\rho}\right)$, then

$$
\left\|R_{n}\right\|^{2}=\sum_{m=0}^{\infty}\left|R_{n}\left(P_{m}\right)\right|^{2}=\sum_{m=0}^{\infty}\left|\int_{-1}^{1} P_{m}(z) d z-\sum_{k=1}^{n} A_{k} P_{m}\left(z_{k}\right)\right|^{2} .
$$

For the space $L^{2}\left(E_{\rho}\right)$, the complete orthonormal sequence can be defined as follows: $P_{m}(z)=2(m+1)^{1 / 2}\left[\pi\left(\rho^{m+1}-\rho^{-m-1}\right)\right]^{-1 / 2} U_{m}(z)$, where $U_{m}(z)=\left(1-z^{2}\right)^{-1 / 2} \times$ $\sin [(m+1) \arccos z], m=0,1, \ldots$. Then

Received July 11, 1966. Revised January 23, 1967.

* The first author is on leave during the 1966-1967 academic year at Brown University, Division of Applied Mathematics, Providence, Rhode Island. 


$$
\left\|R_{n}\right\|^{2}=\sum_{m=0}^{\infty} \alpha(m, \rho)\left|\beta(m)-\sum_{k=1}^{n} A_{k} U_{m}\left(z_{k}\right)\right|^{2},
$$

where $\alpha(m, \rho)=4(m+1) /\left[\pi\left(\rho^{m+1}-\rho^{-m-1}\right)\right] ; \beta(m)=\left[1+(-1)^{m}\right] /(m+1)$. $\left\|R_{n}\right\|$ is a continuous function of the $A_{k}$ and $z_{k}$. The $z_{k}$ are assumed real for the cases considered and this forces the $A_{k}$ to be real also. If we consider the $A_{k}$ and $z_{k}$ as belonging to a compact region in Euclidean $2 n$-space, say, $\left|A_{k}\right| \leqq 1,\left|z_{k}\right| \leqq 1$, $k=1, \cdots, n$, then $\left\|R_{n}\right\|$ has a minimum in the region.

In order to calculate this minimum, we set $\partial\left\|R_{n}\right\|^{2} / \partial A_{k}=0, \partial\left\|R_{n}\right\|^{2} / \partial z_{k}=0$, $k=1, \cdots, n$ and solve the resulting nonlinear system of $2 n$ equations in $2 n$ variables. The equations to be solved are the following:

$$
\begin{aligned}
\sum_{m=0}^{\infty} 2 \alpha(m, \rho)\left(\beta(m)-\sum_{k=1}^{n} A_{k} U_{m}\left(z_{k}\right)\right)\left(-U_{m}\left(z_{j}\right)\right) & =0, \quad j=1, \cdots, n, \\
\sum_{m=0}^{\infty} 2 \alpha(m, \rho)\left(-A_{j}\left(\beta(m)-\sum_{k=1}^{n} A_{k} U_{m}\left(z_{k}\right)\right) U_{m}^{\prime}\left(z_{j}\right)\right) & =0, \quad j=1, \cdots, n .
\end{aligned}
$$

Newton's method is used to solve the system of Eqs. (3). The initial approximations to the $z_{k}$ are the Gaussian base points corresponding to the same value of $n$. The initial approximations to the $A_{k}$ are the $A_{k}{ }^{*}$ which minimize $\left\|R_{n}\right\|$, with the $z_{k}$ fixed as the Gaussian points. The $A_{k}{ }^{*}$ are given in reference [2].

3. Examples and Use of the Tables. Tables of the minimum $\left\|R_{n}\right\|$ and the minimizing $A_{k}$ and $z_{k}$, for various values of $n$ and ellipses $E_{\rho}$, are given in Section 4 .

In this section, we consider the use of the minimum $\left\|R_{n}\right\|$ to estimate the quadrature error $\left|R_{n}(f)\right|$ and we also compare $\left|R_{n}(f)\right|$, using the minimizing $A_{k}$ and $z_{k}$, with $\left|R_{n}(f)\right|$ for known quadrature rules. The upper bound used is $\left|R_{n}(f)\right| \leqq$ ||$R_{n} \| \cdot|| f||$, where $\left.|| f\right|^{2}=\iint_{E_{\rho}}|f(z)|^{2} d x d y$. An upper bound must usually be used for $\|f\|$. One such bound is $M(\pi a b)^{1 / 2}$, where $M$ is the supremum of $|f|$ inside the ellipse $E_{\rho}$. If $f$ is analytic on the ellipse, then $M$ is the maximum of $|f|$ and it occurs on the ellipse.

Example 1. $f$ is analytic on the ellipse $E_{\rho}$ and $M=\sup _{z \in E_{\rho}}|f(z)|=e^{a^{2}}$, for $f(z)=e^{z^{2}}$. Since $b=\left(a^{2}-1\right)^{1 / 2}$, we have $\left|R_{n}\left(e^{z^{2}}\right)\right| \leqq|| R_{n}\|\cdot\| e^{z^{2}} \| \leqq$ $\left\|R_{n}\right\| e^{a^{2}}\left[\pi a\left(a^{2}-1\right)^{1 / 2}\right]^{1 / 2}$. This gives an error bound for $f(z)=e^{2^{2}}$ as a function of $n$ and $a$. For each $n$ we select the value of $a$ from the tables which minimizes this expression. The minimizing values are shown in the table below.

$\begin{array}{ccc}n & \text { Minimizing value of } a & \left\|R_{n}\right\| e^{\omega^{2}}\left[\pi a\left(a^{2}-1\right)^{1 / 2]^{1 / 2}}\right. \\ 2 & 1.50 & 1.26776 \\ 3 & 2.0 & 0.15599 \\ 4 & 2.0 & 0.01290\end{array}$

Example 2. We have $M=a\left(e^{4 b}+e^{-4 b}\right) / 2$ and $\left|R_{n}(z \cos z \sin z)\right| \leqq|| R_{n} \| \cdot M(\pi a b)^{1 / 2}$, for $f(z)=z \cos z \sin z$.

The minimizing values are shown in the table below.

$\begin{array}{ccc}n & \text { Minimizing value of } a & \left\|R_{n}\right\| \cdot M(\pi a b)^{1 / 2} \\ 2 & 1.03 & 2.25136 \\ 3 & 1.03 & 1.78641 \\ 4 & 1.40 & 0.87444\end{array}$


The following table contains comparisons, for specific functions, of minimum norm (MN) quadratures with various known quadratures. Composite rules are used on the functions $1 /\left(1+z^{2}\right)$ and $z \sin z \cos z$ with step-lengths as indicated. The numbers in parentheses indicate the appropriate power of 10 . For each function the same number of base points was used for MN quadratures as for the known quadratures. The Tchebycheff quadratures are the quadratures with equal weights that have the highest polynomial precision [7].
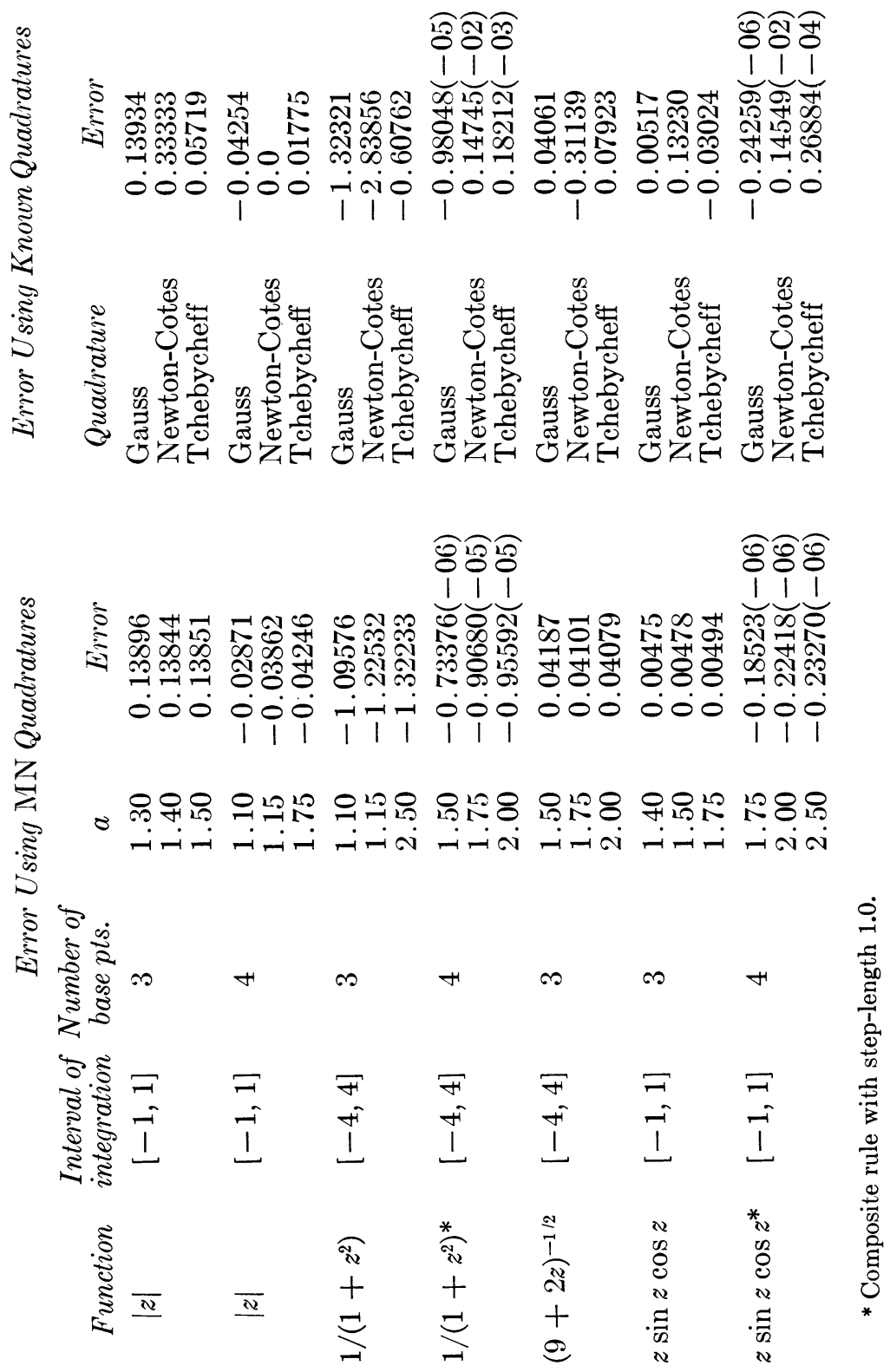
TABLE 1

$$
N=2
$$

\begin{tabular}{c|c|c|c}
\hline$a$ & Base Points & Weights & $\left\|R_{2}\right\|$ \\
\hline 1.03 & 0.5306967015 & 0.5242087319 & 1.7385340982 \\
1.05 & 0.5389972688 & 0.6575665167 & 1.2883434873 \\
1.10 & 0.5519030316 & 0.8369649737 & 0.7293161604 \\
1.15 & 0.5592979275 & 0.9152367390 & 0.4623701537 \\
1.20 & 0.5639700051 & 0.9527037191 & 0.3127386455 \\
1.25 & 0.5671105812 & 0.9720726463 & 0.2213011434 \\
1.30 & 0.5693184230 & 0.9827374321 & 0.1620129721 \\
1.40 & 0.5721257073 & 0.9926623836 & 0.0936211470 \\
1.50 & 0.5737590630 & 0.9965263751 & 0.0582140241 \\
1.75 & 0.5757005520 & 0.9992657692 & 0.0214811009 \\
2.00 & 0.5764713404 & 0.9997914963 & 0.0099094274 \\
2.50 & 0.5770260520 & 0.9999716218 & 0.0028420266 \\
Gauss & 0.5773502692 & 1.0 & \\
\hline
\end{tabular}

TABLE 2

$N=3$

\begin{tabular}{c|l|c|c}
\hline \multicolumn{1}{c|}{ Base Points } & \multicolumn{1}{|c}{ Weights } & \multicolumn{1}{c}{$\left\|R_{3}\right\|$} \\
\hline 1.03 & 0.7434834252 & 0.4015017486 & 1.3800704854 \\
& 0.0 & 0.6003729582 & \\
1.05 & 0.7518233122 & 0.4749670772 & 0.8937754839 \\
& 0.0 & 0.7203543980 & \\
1.10 & 0.7623021863 & 0.5384360267 & 0.3828139543 \\
& 0.0 & 0.8322752623 & \\
1.15 & 0.7669501499 & 0.5530018003 & 0.1960803668 \\
& 0.0 & 0.8630079016 & \\
1.20 & 0.7694119638 & 0.5568194848 & 0.1115324621 \\
& 0.0 & 0.8741094499 & \\
1.25 & 0.7708708741 & 0.5577469582 & 0.0680827745 \\
& 0.0 & 0.8791198738 & \\
1.30 & 0.7718054048 & 0.5578103560 & 0.0437555480 \\
& 0.0 & 0.8818136908 & \\
1.40 & 0.7728879061 & 0.5573648268 & 0.0201919851 \\
& 0.0 & 0.8845753232 & \\
1.50 & 0.7734643431 & 0.5569025309 & 0.0103573945 \\
& 0.0 & 0.8859711882 & \\
1.75 & 0.7740993485 & 0.5562167388 & 0.0026201244 \\
2.00 & 0.0 & 0.8875450457 & \\
& 0.7743365086 & 0.5559146211 & 0.0008661110 \\
2.50 & 0.0 & 0.8881675221 & \\
Gauss & 0.7745019720 & 0.5556895392 & 0.0001506814 \\
& 0.0 & 0.8886207597 & \\
& 0.0 & 0.5555555556 & \\
& & 0.8888888889 & \\
& & &
\end{tabular}


TABLE 3

$N=4$

\begin{tabular}{c|c|c|c}
\hline$a$ & Base Points & Weights & $\left\|R_{4}\right\|$ \\
\hline 1.03 & 0.8434055237 & 0.3019737608 & 1.0316186099 \\
& 0.3283257294 & 0.5308958137 & \\
1.05 & 0.8495395476 & 0.3342347346 & 0.5717864022 \\
1.10 & 0.3319553911 & 0.5977818841 & \\
& 0.8557804260 & 0.3503185979 & 0.1845142780 \\
1.15 & 0.3357683847 & 0.6390052212 & \\
& 0.8580390968 & 0.3512050953 & 0.0770467932 \\
1.20 & 0.3372551809 & 0.6463753888 & \\
& 0.8591144634 & 0.3506375343 & 0.0371216097 \\
1.25 & 0.3380354752 & 0.6486767179 & \\
& 0.8597141460 & 0.3500424633 & 0.0196398593 \\
1.30 & 0.3385155033 & 0.6497312377 & \\
& 0.8600844267 & 0.3495766937 & 0.0111137456 \\
1.40 & 0.3388388676 & 0.6503397858 & \\
& 0.8605008925 & 0.3489647267 & 0.0041087299 \\
1.50 & 0.3392399970 & 0.6510207626 & \\
& 0.8607177992 & 0.3486096510 & 0.0017410793 \\
1.75 & 0.3394709812 & 0.6513871622 & \\
2.00 & 0.8609535029 & 0.3481958730 & 0.0002973320 \\
& 0.3397457245 & 0.6518039877 & \\
2.50 & 0.8610408334 & 0.3480351680 & 0.0000716323 \\
Gauss & 0.3398553575 & 0.6519648209 & \\
& 0.8611015909 & 0.3479209825 & 0.0000075609 \\
& 0.3399345844 & 0.6520790173 & \\
& 0.8611363116 & 0.3478548451 & \\
& 0.3399810436 & 0.6521451549 & \\
\hline
\end{tabular}

4. Tables. Tables 1,2 , and 3 list the values of the quadrature weights $A_{k}$ and base points $z_{k}$, and the corresponding values obtained for $\left\|R_{n}\right\|$ from Eq. (2), for $n=2,3,4$, respectively. The minimizing values of the $z_{k}$ are symmetric; hence, only the nonnegative ones are listed. The weights obtained for symmetric base points are equal and so only those weights corresponding to nonnegative base points are listed.

5. Conclusions. For the numerous functions tested minimum norm quadratures were, overall, comparable in accuracy to Gaussian quadratures and better than Newton-Cotes and Tchebycheff quadratures. It is generally the case that composite rules must be used to achieve sufficient accuracy in a practical problem and the quadratures of the function $z \sin z \cos z$ given in Section 3 illustrate the use and accuracy of a composite minimum norm quadrature. It might be noted that the MN rules do not integrate constants exactly and so those theorems requiring the sum of the weights to equal the length of the interval do not apply.

The MN quadratures have interesting asymptotic properties, both as $\rho \rightarrow \infty$ and as $n \rightarrow \infty$. From Tables 1, 2 and 3 it can be seen numerically that the weights and base points of the MN quadratures seem to approach the weights and base 
points of the Gaussian quadratures with the same number of points. Valentin [8] has proved a similar result and his proof can be altered to prove the above conjecture, the details of which will appear in a future paper.

6. Acknowledgements. This research was supported in part by the National Science Foundation under Grant GP 5906; the second author was also supported by an NDEA Title IV Fellowship at the University of Utah. The authors wish to thank the University of Utah Computer Center for the use of their IBM 7044. The authors also wish to thank the referee for his helpful criticisms.

Department of Mathematics

University of Utah

Salt Lake City, Utah

1. R. E. Barnhill, "Complex quadratures with remainders of minimum norm," Numer. Math., v. 7, 1965, pp. 384-390. MR 32 \#497.

2. R. E. BARNHILl \& J. A. WixOM, "Quadratures with remainders of minimum norm. I," Math. Comp., v. 21, 1967, pp. 66-75.

3. P. J. DAvis, "Errors of numerical approximation for analytic functions," J. Rational Mech. Anal., v. 2, 1953, pp. 303-313. MR 14, 907.

4. P. J. DAvis, "Errors of numerical approximation for analytic functions," Survey of Numerical Analysis, McGraw-Hill, New York, 1962, pp. 468-484. MR 24 \#B1766.

5. P. J. Davis, Interpolation and Approximation, Blaisdell, New York, 1963. MR 28 \#393.

6. P. Davis \& P. Rabinowitz, "On the estimation of quadrature errors for analytic functions," MTAC, v. 8, 1954, pp. 193-203. MR 16, 404. \#2008.

7. V. I. Krylov, Approximate Calculation of Integrals, Macmillan, New York, 1962. MR 26

8. R. A. Valentin, Applications of Functional Analysis to Optimal Numerical Approximation for Analytic Functions, Ph.D. thesis, Brown University, Providence, R. I., 1965.

9. H. Yanagihara, "A new method of numerical integration of Gaussian type," Bull. Fukuoka Gakugei Univ. III, v. 6, 1956, pp. 19-25. (Japanese) MR 26 \#5729.

10. R. E. BARnhill, Numerical Contour Integration, U. S. Army Math. Research Center Report No. 519, 1964.

11. H. S. WILF, "Exactness conditions in numerical quadrature," Numer. Math., v. 6, 1964, pp. 315-319. MR 31 \#4178.

12. P. J. Davis \& R. Rabinowitz, Numerical Integration, Blaisdell, New York, 1967. 